\title{
Les conflits d'intérêts dans le domaine des produits et de technologies de santé : état des lieux et recommandations
}

\author{
Jean-Paul Demarez ${ }^{1}$, Christian Funck-Brentano ${ }^{2}$, Mathieu Molimard ${ }^{3}$ et les participants à la table ronde $N^{\circ} 4$ \\ de Giens XXVII* \\ 1 Département de Pharmacovigilance, Laboratoire Pierre-Fabre, Boulogne-Billancourt, France \\ 2 AP-HP Pitié-Salpêtrière, UPMC, Département de Pharmacologie, Paris, France \\ 3 CHU et Université de Bordeaux, Département de Pharmacologie, Bordeaux, France
}

Texte reçu le 20 mars 2012 ; accepté le 4 juin 2012

\section{Mots clés :}

liens d'intérêts ; experts ; impartialité

\begin{abstract}
Résumé - La gestion des conflits d'intérêts dans le domaine des produits et des technologies de santé est devenue un enjeu majeur pour tous les acteurs de santé. La table ronde $\mathrm{N}^{\circ} 4$ de Giens 2011 a fait des propositions concrètes pour clarifier et optimiser cette gestion des conflits d'intérêts.

Les conflits d'intérêt ne peuvent pas être définis par les personnes consultées ou demandeuses de fonds puisque chaque institution, privée ou publique, émettant des appels à projets, ou demandeuse d'avis, d'analyses ou d'expertises en matière de santé a des niveaux variables de sa définition de ce qu'est un conflit dans le contexte particulier de la proposition ou demande particulière qu' elle émet. En revanche, chaque individu a des liens d'intérêt qui peuvent et doivent être explicitement déclarés. Ces liens sont bien plus diversifiés que ce qu'il est commun de trouver dans les déclarations d'intérêt des grands organismes liés à la santé et ne se limitent pas aux liens matériels et financiers entre entreprises et individus. Ces déclarations sont par ailleurs lourdes à gérer du fait de leur multiplication.

La recommandation de la table ronde est que chaque individu liste de manière explicite dans une déclaration unique des liens d'intérêt (DULI), l'ensemble de ses liens d'intérêts. Cette déclaration serait mise à jour en continu et accessible en ligne. Chaque institution pourrait ainsi déterminer si les liens déclarés constituent, ou non, un conflit pour la mission demandée. Les conditions génératrices de conflit seraient affichées a priori par chaque institution de telle sorte qu'un individu consulté puisse se récuser pour la mission proposée.

D'autres mécanismes opérationnels de gestion des conflits ont été proposés.
\end{abstract}

Abréviations : voir en fin d'article.

\section{Cadre et définitions}

Les conflits d'intérêt dans le monde de la santé constituant une préoccupation tant pour l'opinion que pour les pouvoirs publics, les membres de la présente table ronde (TR) avaient pour but de constituer un état des lieux et de proposer des recommandations dans le domaine plus précis des produits et technologies de santé.

Les professionnels, médecins ou pharmaciens, concernés par le domaine des produits de santé, sont, par les différentes positions et rôles sociaux qu'ils sont susceptibles d'occuper ou de remplir, placés dans des situations susceptibles de faire naître des conflits d'intérêt. Ils peuvent être tout à la fois en relation de soins avec des patients, agents publics ou en exercice libéral, enseignants à l'université ou dans les formations post-universitaires, chercheurs au sein d'établissements publics ou privés, conseils ou investigateurs à la demande d'entreprises de la pharmacie, experts missionnés par des commissions publiques, membres de ces commissions...

Toutes ces activités sociales se traduisent nécessairement par des liens d'intérêts. Les premiers qui viennent à l'esprit pour beaucoup de raisons pratiques comme symboliques sont les liens d'argent. Dans la plupart des situations évoquées ci-dessus, le professionnel peut recevoir, en échange de sa prestation une contrepartie monétaire dont la diversité d'appellation, salaire, traitement, honoraires, subvention, prime, etc. traduit la diversité de la nature des liens. Mais la question des conflits d'intérêts ne se limite pas

\footnotetext{
* Pour la liste des participants, voir en fin d'article.
} 
aux seuls aspects économiques. Les professionnels concernés ont, comme la plupart, d'autres liens susceptibles d'influer sur leurs choix, consécutifs à leur vie familiale, affective, à leurs appartenances associative, politique ou syndicale, philosophique comme religieuse, aux connivences intellectuelles ou professionnelles qu'ils ont pu établir, à leur cursus ou leurs espérances de carrière, aux hiérarchies dans lesquelles ils s'intègrent... bref, à tout ce qui découle de la trajectoire humaine.

De tels liens sont propres à la vie en société et très peu nombreux sont ceux qui en sont exempts ou qui peuvent s'en exempter.

Certains de ces liens peuvent, à telle ou telle occasion, risquer de faire naître voire créer effectivement un « conflit d'intérêts ».

Les définitions proposées pour cette expression sont nombreuses. Les membres de la table ronde se sont accordés pour dire qu'un conflit d'intérêt est cette situation dans laquelle le professionnel n'agirait pas ou pourrait être soupçonné de ne pas agir en fonction des intérêts qu'il est socialement censé défendre ou prendre en charge, mais dans le but d'avantager d'autres intérêts, les siens ou ceux d'un tiers.

Cette définition est évidemment à replacer dans le contexte des produits et technologies de santé, objet de la présente analyse.

\section{Particularités du présent contexte}

Les produits de santé font l'objet, préalablement à leur exploitation commerciale, de décisions successives des autorités ou des organismes compétents relatifs à leur autorisation de mise sur le marché, à leur prise en charge par les organismes d'assurancemaladie ou les mutuelles, à leurs conditions d'utilisation, aux diverses recommandations orientant les stratégies thérapeutiques dans lesquels ils pourraient s'inclure...

Ces différentes décisions sont prises après consultation de commissions et avis d'experts, les membres des commissions et les experts missionnés étant choisis parmi les professionnels connus pour avoir les connaissances leur permettant d'éclairer utilement les décideurs.

Le vocabulaire courant utilise le terme « indépendant » pour définir le principal qualificatif caractérisant le «bon » expert. Ce terme s'avère impropre, compte tenu de l'existence des liens multiples dont il est fait état ci-dessus. Un expert ne peut en réalité s'en affranchir et donner les garanties qu'il s'en est affranchi.

Plutôt qu'indépendant, il est attendu d'un expert qu'il soit impartial.

Dans l'intérêt général comme dans l'intérêt particulier des malades, les expertises réalisées ne doivent pas être biaisées par des considérations ou intérêts étrangers à la mission de l'expert, ni les décisions qui seront prises à l'issue des expertises entachées de partialité.

Les pouvoirs publics se sont depuis longtemps préoccupés de cette question de conflits d'intérêts notamment chez les membres des commissions consacrées aux médicaments et aux produits de santé ainsi qu'aux experts du domaine en mettant en place un système de déclarations régulières. Le principe de cette déclaration paraissait suffisant pour détecter les conflits et tirer les conséquences de leur existence éventuelle. L'Agence française de sécurité sanitaire des produits de santé (Afssaps, devenue l'Agence nationale de sécurité du médicament et des produits de santé [ANSM], la Haute autorité de santé (HAS), l'Institut national du cancer (INCa), par exemple, ont mis en place, outre des questionnaires spécifiques, des normes internes censées permettre un contrôle et une gestion adaptée. Toutefois, il résulte de tout ceci un ensemble hétérogène, compliqué, redondant, conduisant les experts consultés par l'une ou l'autre de ces structures à des exercices inutilement répétitifs. L'hétérogénéité de l'ensemble ne plaide pas en faveur de son efficacité.

La finalité de la présente table ronde est de définir une déclaration unique des liens d'intérêt, déclaration unique (ou universelle ?) des liens d'intérêts (DULI), simplifiant et améliorant les procédures déclaratives, et de proposer des règles d'utilisation de cette déclaration des liens d'intérêts par les demandeurs d'avis et d'expertises afin de déterminer si ces liens constituent ou non des conflits d'intérêts et comment ils convient de les gérer si de tels conflits existent.

La connaissance des liens propres aux experts mais également propres aux décideurs doit permettre d'éviter que les uns comme les autres soient sous l'influence de conflits d'intérêts au moment de remplir la mission ou de tenir le rôle qui leur incombe.

L'objectif d'intérêt général poursuivi ici dépasse largement le champ des autorisations administratives et de la prise en charge de tel ou tel produit de santé pour englober les dépenses de santé, le progrès thérapeutique et le bon usage des médicaments, l'information des professionnels et celle du public et la définition des politiques de santé.

La question des conflits d'intérêt est par conséquent susceptible de s'étendre à tous les intervenants y compris aux journalistes de la presse spécialisée ou généraliste participant à l'information des professionnels de santé ou du public.

\section{Les obligations de l'expert}

Si nous recentrons la présente analyse sur l'expert missionné par telle ou telle institution, étant admis le caractère normal des liens d'intérêts, il est possible de retenir quelques considérations pratiques. L'expert est le premier juge, en conscience, de l'éventualité de l'existence d'un conflit entre l'un des liens d'intérêt dont il est bénéficiaire et l'intérêt que l'institution le charge de défendre, nul n'étant obligé d'accepter une mission d'expertise.

Mais là ne doit pas s'arrêter l'examen. Il incombe à l'expert de déclarer lui-même à l'institution qui envisage, voire même qui serait susceptible d'envisager de le missionner, les liens financiers directs comme indirects, principaux ou accessoires, mais également tous les autres liens qui lui apparaissent de nature à conduire 
à une situation de conflit d'intérêts, dont l'inventaire pourra figurer sous une forme à la fois standardisée et exhaustive (voir plus loin).

Il appartient ensuite à la structure concernée, au moment d'une désignation d'expert, après avoir pris connaissance des liens déclarés par les personnes susceptibles d'être missionnées, d'identifier, le cas échéant, un conflit, positif ou négatif, majeur ou mineur, et d'en tirer les conséquences qui s'imposent, après avoir éventuellement permis à un impétrant de clarifier sa situation, en application du principe du contradictoire.

Les déclarations faites par le professionnel postulant au rôle d'expert doivent être accessibles, publiques, soumises à une actualisation dès lors qu'une modification implique substantiellement la liste des liens précédemment déclarés.

La forme utilisée pour des déclarations individuelles des liens d'intérêt doit assurer leur lisibilité et permettre de façon simple d'identifier que certains liens constituent ou peuvent constituer un conflit d'intérêt dans le contexte particulier de la mission demandée.

Il est à souligner qu'un même lien d'intérêt peut se présenter comme conflictuel dans une situation donnée mais pas dans une autre circonstance.

Un lien d'intérêt peut induire chez un expert la tentation d'influer en faveur d'un intérêt tiers, ce que nous appellerons un conflit positif. Un lien peut permettre également à un expert de nuire à un intérêt tiers, cas de figure du conflit négatif. Tous les liens d'intérêt ne présentent pas le même impact sur la situation de l'expert et l'on peut identifier dans la liste des liens la possibilité de conflits majeurs comme de conflits mineurs.

Le caractère exhaustif de la déclaration de liens d'intérêt va éventuellement conduire le déclarant à prendre en considération des liens le concernant dans sa vie privée. Il convient qu'une rubrique lui permette de le faire en préservant son intimité tant visà-vis des destinataires de la déclaration que d'éventuels lecteurs de la déclaration, celle-ci étant publique.

S'agissant du cas particulier des experts se prononçant dans le cadre ou à la demande des commissions consultatives compétentes en matière de produits ou de technologie de santé, les membres de la table ronde ont émis cinq propositions :

- constitution d'une déclaration unique par chaque expert de ses liens d'intérêts sur une adresse Internet au moyen d'un document uniformisé sous forme de tableau DULI dont les rubriques renseignement la nature, le type, la durée et le champ du (ou des) lien(s) [tableau I] ;

- examen de ces liens par l'institution intéressée préalablement à la désignation de l'expert, examen pouvant entraîner sa récusation. L'éventualité de cette récusation peut conduire l'institution à aller plus avant dans le questionnement et doit ménager l'application du principe du contradictoire ;

- désignation, pour une même mission, de plusieurs experts en concurrence. La collégialité paraît être un excellent facteur d'impartialité ;

- constitution par les différentes institutions publiques concernées d'une rubrique questions/réponses relative aux conflits d'intérêts, accessible aux experts, et leur permettant d'avoir des éléments de réflexion conduisant à leur éventuelle déportation sur un dossier particulier. Cette rubrique peut prendre la forme d'une collection de « jurisprudence », évoquant des cas pratiques ;

- sanction des experts volontairement oublieux. Les déclarations incomplètes, que ce soit involontairement ou volontairement, constituent un motif d'annulation par la juridiction administrative des décisions ou des actes faisant suite à une expertise ainsi entachée.

Il convient, pour favoriser la prise en compte par les professionnels de santé concernés des problématiques inhérentes aux liens d'intérêts, que des enseignements, des formations et des évaluations soient mises en place, tant par les pouvoirs publics, les sociétés savantes que par les associations représentatives.

\section{La DULI}

La DULI (tableau I) permet de déclarer tout lien économique, salaire, honoraires de consultant ou d'expert, d'investigateur participant à une recherche médicale, défraiement ou prise en charge des frais d'un membre de comité ou de commission, d'un intervenant à un congrès, d'un conférencier, intérêts versés à un actionnaire ou à un titulaire de droits sur un brevet..., notamment..., car le tableau DULI semble être « tout terrain » à cet égard.

Mais, elle a été conçue pour permettre d'identifier également tout lien non financier, direct ou indirect, et nous avons pu au début de cette analyse évoquer la grande diversité existant en la matière.

Outre sa consultation avant désignation d'un expert, la consultation de la DULI pourra se faire au début de toute séance d'une commission, s'agissant des membres présents, par la vérification de liens particuliers comme par l'affichage à l'attention de tous les participants des liens identifiés comme conflictuels.

Elle peut être utilisée par tout expert pressenti ou aspirant à l'être, tout professionnel de santé, tout décideur en la matière, tout membre d'une société savante ou d'une association de malades, tout journaliste intervenant dans le domaine à destination des institutions publiques, des sociétés savantes, des organes de presse, du public intéressé en général.

La DULI doit permettre l'identification de la nature (positif comme négatif) et du niveau (majeur ou mineur) du conflit d'intérêt potentiel ou avéré avant que soit prise la position de l'institution.

La plupart du temps, la déportation de l'expert par lui-même ou sa récusation par l'institution apporte la solution à la question posée. Il est toutefois des disciplines scientifiques ou des domaines, notamment celui des maladies orphelines, où le nombre d'experts disponibles s'avère particulièrement restreint. L'alternative oppose le risque de mauvaise qualité scientifique de l'expertise au risque d'expertise biaisée. Cet état de fait ne manquera pas de retentir sur le niveau d'exigence relatif à l'acceptation des conflits d'intérêts, ce 


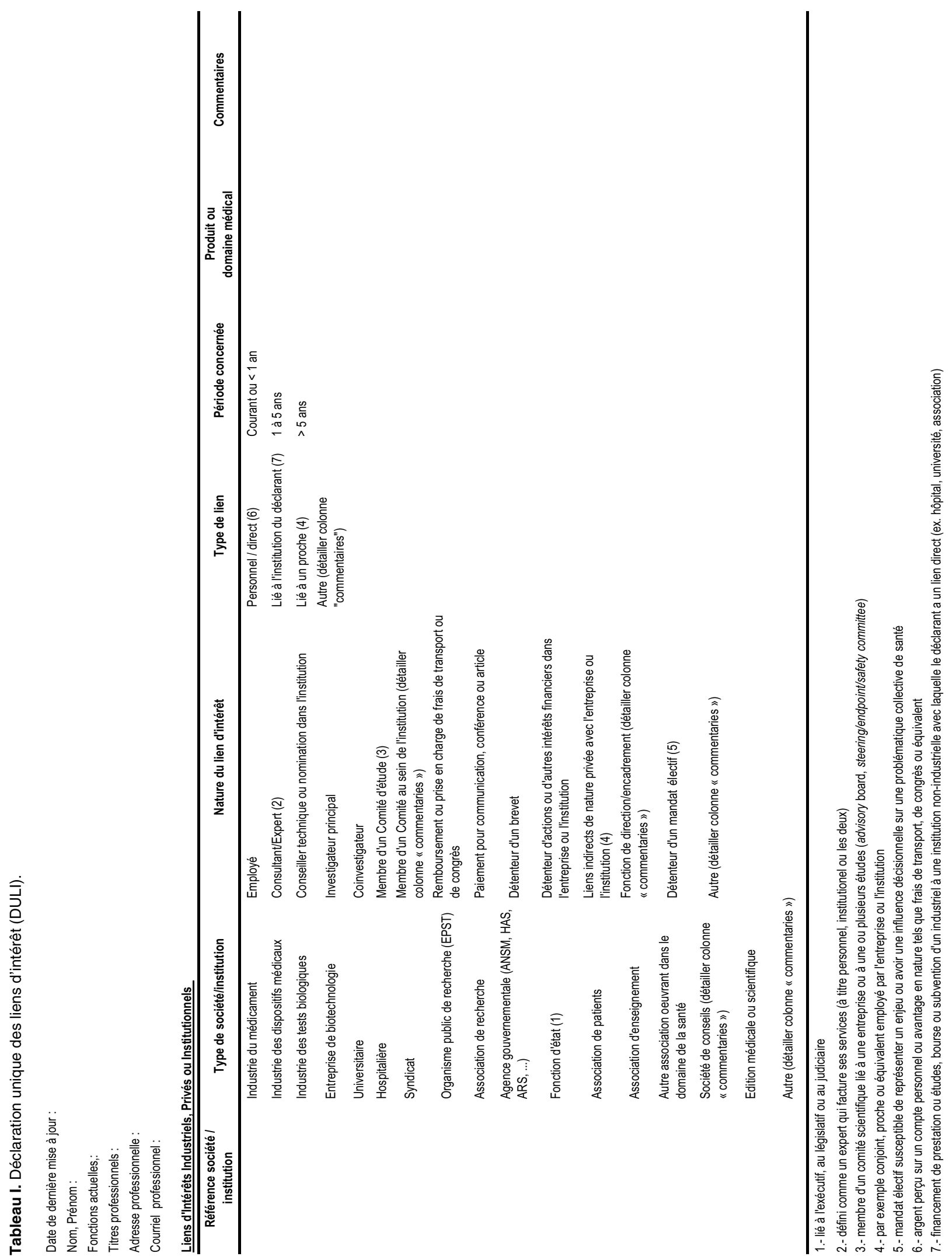


niveau d'acceptation apparaissant inversement proportionnel au nombre d'experts compétents susceptibles d'être missionnés. Dans ces situations, il appartient à l'institution souhaitant l'expertise d'organiser les conditions d'intervention des experts porteurs de conflits d'intérêts. Ces experts peuvent, par exemple, rendre leur analyse, mais être exclus de toute intervention dans la décision finale.

À l'intérieur du cadre commun et de la grille d'interprétation commune, tant aux experts qu'aux institutions, il appartient à chaque institution de faire ses choix de manière transparente, et, le cas échéant de les motiver dès lors qu'ils comportent l'acceptation de conflits d'intérêts.

\section{Sanctions des liens non déclarés}

Étant entendu que les déclarations ont vocation à être complètes, sincères et véritables, et qu'une déclaration affichant une totale absence de liens d'intérêts ne saurait être crédible, il est possible de considérer que l'expert ayant déclaré l'intégralité des liens dont il est l'objet est dans la situation la plus claire vis-à-vis des pouvoirs publics : «liens déclarés - expert protégé ».

Il peut toutefois advenir qu'à l'occasion d'une inspection, d'une contestation de la décision prise, voire d'une dénonciation, apparaissent des manquements dans la déclaration faite par l'expert, ou un défaut significatif d'actualisation de cette déclaration.

La bonne foi de l'expert peut être reconnue et le manquement résulter d'une omission, d'une étourderie, d'une erreur...

Il n'en est pas moins certain, les faits étant avérés, que l'expert s'expose ainsi à une sanction disciplinaire prise à son encontre par l'ordre professionnel dont il dépend, le caractère involontaire de la faute n'étant pas exonérateur de responsabilité.

Une déclaration incomplète résultant d'une manipulation volontaire de la réalité expose elle aussi à des sanctions disciplinaires, mais est parallèlement constitutive d'une infraction pénale exposant à une sanction de même nature.

Les faits peuvent être qualifiés de faux en écriture privée, de prise illégale d'intérêt, voire même, si les éléments d'information disponibles le permettent, de complicité d'escroquerie.

Une décision administrative prise à l'aide d'avis d'experts dont la régularité est ainsi contestable, que ce soit de façon involontaire ou volontaire, est susceptible d'être annulée par la juridiction administrative en raison d'une violation des règles légales relatives à l'élaboration des actes administratifs.

\section{Experts et évaluateurs}

Les décisions prises par les autorités compétentes en matière de produits et de technologie de santé le sont après un travail d'analyse préparatoire, concernant ses dimensions techniques ou scientifiques.
Deux catégories de personnes sont amenées à participer à ces travaux préparatoires, des évaluateurs internes, membres salariés des dites autorités compétentes, des experts externes choisis en raison de leur réputation dans le domaine concerné. Les experts externes appartiennent la plupart du temps au cadre hospitalier ou hospitalo-universitaire.

Il va de soi que les évaluateurs internes ne peuvent avoir de liens d'intérêts économiques (les seuls facilement individualisables) avec d'autres organisations que l'administration qui les emploie, sauf dérogation exprès de leur hiérarchie.

Les évaluateurs sont en miroir des avantages et des inconvénients des experts : ils n'ont pas de lien économique susceptible de biaiser leur travail, avantage vis-à-vis des experts qui ont cette possibilité.

Les experts le sont devenus du fait d'une expérience acquise au cours d'une carrière scientifique parfois avancée, les évaluateurs sont, eux, la plupart du temps des juniors. Une fois leur expérience acquise, ils peuvent être tentés de la valoriser dans d'autres structures que leur administration d'origine.

Mais si les évaluateurs peuvent également espérer construire un profil de carrière à partir de leur poste initial qui leur assure un traitement, il n'en est pas de même pour les experts externes. En effet, les activités d'expertise ne sont pas prises en considération dans le cursus de professionnels qui s'y prêtent, et s'exercent le plus souvent sans contrepartie réelle du travail effectué de la part de l'Administration qui a recours à eux.

Devant cette situation paradoxale, les membres de la table ronde $\mathrm{N}^{\circ} 4$ estiment, en conclusion de leurs réflexions :

- qu'il convient, d'une part, que les évaluateurs internes puissent développer leur expérience au sein de leur administration avec des niveaux de rémunération propres à retenir des seniors, tout lien d'intérêt économique extérieur leur étant interdit. Ceci contribuera à renforcer l'évaluation interne ;

- de l'autre, qu'il est nécessaire que la situation de bénévolat illusoire dans laquelle s'exerce l'expertise externe disparaisse. L'activité d'expertise externe doit être valorisée et intégrée au profil de carrière des intéressés, parallèlement à la possibilité de liens économiques. L'influence de ces liens sur les activités expertales éventuelles sera d'ailleurs tempérée par la consultation de leur DULI.

\section{Participants.}

Marion Bamberger (Bristol Myers Squibb), François Bassompierre (AP HP, DRCD, Paris), Paul Benkimoun (journal Le Monde, Paris), Driss Berdaï (CHU, Bordeaux), Jean-François Bergmann (Hôpital Lariboisière, Paris), Pierre-Henri Bertoye (ANSM, ex-Afssaps), Nathalie Billon (Laboratoire Sanofi-Aventis), Frédérique Brudon (Fédération des spécialités médicales, Villeurbanne), Gaëlle Courtois (AP HM, Marseille), Claudie Damour-Terrasson (journal Edimark, Paris), Rima De Sahb (Laboratoires MSD-Chibret), Muguette Depardon (HAS, Saint Denis la Plaine), François Faurisson (Eurordis), Alain Francillon (Laboratoire Novartis), Jean Gardette (ANSM, ex-Afssaps), François Garnier 
(Laboratoire Pfizer), Véronique Lamarque-Garnier (Laboratoire Pfizer), Catherine Lassale (Leem), Claire Le Jeunne (AP HP, Hôtel Dieu, Paris), François Montastruc (CHU, Toulouse), Nicholas Moore (CHU, Bordeaux), Joël Moret-Bailly (Université de Lyon, Sainte Foy lès Lyon), Christine M'Rini (Institut Mérieux), Marc Rodwin (Suffolk University Law School, Boston, USA), Myriam Zylberman (Laboratoire Lilly France).

Conflits d'intérêts. Aucun.

Abréviations. Afssaps : Agence française de sécurité sanitaire des produits de santé ; ANSM : Agence nationale de sécurité du médi- cament et des produits de santé ; DULI : déclaration unique (ou universelle) des liens d'intérêts ; HAS : Haute autorité de santé ; INCa : Institut national du cancer.

Correspondance et offprints: Jean-Paul Demarez, Département de Pharmacovigilance, Laboratoire Pierre-Fabre, 45 place Abel Gance, 92654 Boulogne Cedex, France.

E-mail : jean.paul.demarez@pierre-fabre.com 